\title{
Pluridisciplinarité et naissance de l'histoire africaine de langue française
}

Les Cahiers d'Études africaines 1960-1976

Multidisciplinarity Approach and Birth of Francophone African History. The

Cahiers d'Études africaines 1960-1976

\section{Catherine Coquery-Vidrovitch}

\section{OpenEdition}

Journals

Édition électronique

URL : https://journals.openedition.org/etudesafricaines/16231

DOI : 10.4000/etudesafricaines. 16231

ISSN : $1777-5353$

\section{Éditeur}

Éditions de l'EHESS

\section{Édition imprimée}

Date de publication : 20 novembre 2010

Pagination : $545-556$

ISBN : 978-2-7132-2252-8

ISSN : 0008-0055

\section{Référence électronique}

Catherine Coquery-Vidrovitch, « Pluridisciplinarité et naissance de l'histoire africaine de langue française », Cahiers d'études africaines [En ligne], 198-199-200 | 2010, mis en ligne le 02 janvier 2013 consulté le 22 avril 2022. URL : http://journals.openedition.org/etudesafricaines/16231 ; DOI : https:// doi.org/10.4000/etudesafricaines.16231

Ce document a été généré automatiquement le 22 avril 2022.

(c) Cahiers d'Études africaines 


\section{Pluridisciplinarité et naissance de l'histoire africaine de langue française}

Les Cahiers d'Études africaines 1960-1976

Multidisciplinarity Approach and Birth of Francophone African History. The

Cahiers d'Études africaines 1960-1976

Catherine Coquery-Vidrovitch

1 Ayant commencé à étudier l'Afrique en 1960, l'année même de la naissance de la revue ${ }^{1}$, il m'a paru souhaitable de reconstituer, un demi-siècle plus tard, ce que les Cahiers ont alors apporté et comment on peut l'apprécier aujourd'hui. Étant historienne, je vais évidemment m'appuyer plutôt sur cette discipline, même si la revue, introduite en 1960 par l'historien Fernand Braudel, alors président de la $\mathrm{VI}^{\mathrm{e}}$ section de l'École pratique des hautes études (EPHE) (devenue l'École des hautes études en sciences sociales [EHESS] en 1975), a d'emblée affirmé l'originalité de l'approche pluridisciplinaire du domaine « africaniste ». Je me suis attachée à la période 1960-1976, précisément parce que c'est, entre autres, celle de la « construction » de la discipline « histoire de l'Afrique », tel que le reconnaît le dernier numéro que j'analyse ici, le numéro double 61-62 du volume $\mathrm{XVI}^{2}$. C'est effectivement un des points résultant de l'examen auquel je me suis livrée: l'histoire africaine française a connu droit de cité grâce aux (et par les) Cahiers d'Études africaines.

2 Ce qui ressort de ces premières années, c'est d'abord que la revue est créée par et pour les directeurs d'études qui viennent de se constituer en une aire culturelle : l'Afrique noire, comme l'on disait alors, pour la différencier de l'Afrique du Nord traditionnellement désignée du terme de Maghreb (hérité de l'arabe) dans l'historiographie coloniale française qui était en train de prendre fin. Ce sont les différents directeurs en poste qui écrivent les articles, choisissent et organisent les numéros qui vont se succéder de façon apparemment assez aléatoire ${ }^{3}$. Ce sont d'ailleurs les seuls rédacteurs des premiers numéros: dans le numéro 1 figurent Georges 
Balandier et Paul Mercier, tous deux sociologues, Denise Paulme, anthropologue spécialiste des femmes, Henri Brunschwig, historien. Gilles Sautter, le géographe, prend place dans le numéro 2. Le directeur de la revue, le linguiste Pierre Alexandre (spécialiste des langues bantoues), introduit le numéro 6 (1961). On trouve en revanche peu trace de l'autre linguiste qui a marqué l'époque et le centre, le spécialiste de la langue peule Pierre-Francis Lacroix ${ }^{4}$. Ce sont ces mêmes spécialistes qui ont créé un enseignement collectif original destiné à former à la pluridisciplinarité africaniste les jeunes chercheurs encore ignorants du domaine que nous étions alors, la plupart en passe d'être recrutés à l'EPHE (c'était mon cas) ${ }^{5}$, ou jeunes chercheurs plus ou moins en quête d'un poste stable (cas de Henri Moniot, bientôt maître-assistant à la Sorbonne, ou de Claude Meillassoux recruté par le CNRS). À l'image de ce cycle de cours voulu par l'équipe, la direction de la revue est apparemment collégiale, et les noms de quelquesuns des directeurs de recherche (Pierre Alexandre, Henri Brunschwig, Gilles Sautter) reviennent fréquemment. La plus assidue à publier des articles est la sociologue Denise Paulme (qui, la même année 1960, a dirigé un épais numéro de la revue Présence africaine sur les femmes africaines). Le plus absent des auteurs est Georges Balandier dont le nom n'apparaît qu'incidemment, même si, d'évidence, il est l'inspirateur d'une rubrique lancée dès le numéro 1 et qui ne disparaît qu'en 1965 : «Sociologie politique de l'Afrique noire" (dernière mention dans le numéro 19). Georges Balandier ne réapparaît vraiment qu'avec la présentation d'un numéro spécial relativement tardif mais qui, à sa parution, créa l'événement par le travail conjugué de nombre de ses disciples: «Les relations de dépendance personnelle », (numéro 35, 1969), avec les interventions, entre autres, de Roger Botte, Claudine Vidal et Marc Le Pape, PierrePhilippe Rey, Marc Augé, Claude-Hélène Perrot... Une explication est sans doute à trouver dans le fait que, depuis longtemps déjà, Georges Balandier écrivait dans les Cahiers internationaux de sociologie, tandis que pour d'autres disciplines (et peut-être surtout pour l'histoire), aucune revue généraliste n'était disposée à accueillir un article sur un domaine précisément réputé « sans histoire » : l'Afrique subsaharienne.

3 La revue faisait à l'évidence feu de tout bois, avec des chercheurs peu nombreux, souvent des jeunes attirés par ce pôle alors unique dans l'univers académique français : unique par sa nouveauté, car d'autres revues bien plus anciennes existaient. Mais, exceptée la revue Présence africaine créée à Paris par Alioune Diop dès 1947, elles allaient avoir encore pendant un certain nombre d'années du mal à se dégager de leur passé colonial proche : celui de la Société des Africanistes d'une part, de la Société française d'Histoire d'Outre-mer d'autre part, ou des Cahiers d'Outre-mer pour la géographie. Les Cahiers d'Études africaines des premières années ne font pas ou très peu appel à des compétences hors de leur cercle proche, marquant ainsi leur différence. C'est sans doute la raison pour laquelle les numéros thématiques sont rares au début de l'existence de la revue : sauf exception, il n'y avait pas encore assez de matière, dans la première décennie, pour en faire état tout au long d'un numéro. Ainsi la confection du numéro 5 (1961) consacré à l'Éthiopie a-t-elle été confiée au spécialiste Joseph Tubiana ; le numéro 26 (1967) dirigé par Gilles Sautter est consacré à l'Afrique équatoriale et centrale (des deux Congos) ; il y publie notamment son mémorable texte (90 pages) sur l'histoire meurtrière de la construction du chemin de fer Congo-Océan (1921-1934). Mais il faudra attendre le numéro de Balandier, cité supra sur les dépendances personnelles (1969), pour trouver le premier numéro vraiment thématique. Il est le signal d'une nouvelle étape: les directeurs de recherche "maitres à bord" ont désormais tous formé une école de disciples dont ils peuvent rassembler les travaux sur 
un de leurs thèmes privilégiés (on avait vu apparaître Jean-Loup Amselle en 1969 [numéro 34] et Jean Copans en 1971 [numéro 43]).

Dès lors il y aura, sans périodicité particulière, à plusieurs reprises des numéros partiellement ou entièrement thématiques. L'une des plus actives en ce domaine fut Denise Paulme, qui lance en 1970 un numéro sur les rituels religieux (numéro 38), puis successivement deux numéros sur la littérature orale et le folklore: le premier en collaboration avec Christiane Seydou et la section littérature du CNRS (numéro 45, 1972), le second sur les contes (numéro 60,1975). Gilles Sautter organise un numéro sur les civilisations rurales (numéro 47,1972 ) et un autre, qui fera tout autant date, en collaboration avec Paul Mercier sur les villes (numéro 51, 1973) : on y trouve (comme désormais dans les autres numéros) les noms de nombreux jeunes chercheurs qui se préparent à dominer la génération suivante: René Gouellain, Philippe Haeringer, Annick Osmont, Jean-Marie-Gibbal, Marc Vernière ${ }^{6} . .$. Quant à Claudine Vidal, prenant le relais du «maître » Balandier, elle consacre un numéro aux relations de dépendances personnelles au Rwanda dès 1974 (numéro 53).

5 On sent donc deux impératifs : le premier est d'aborder des sujets originaux de façon nouvelle. De ce point de vue, quel florilège! Un des articles majeurs de l'époque (on était en pleine remise en cause "poststalinienne » des enseignements marxistes) fut, dès le numéro 12 (1963), celui, révolutionnaire, du jeune anthropologue marxiste Claude Meillassoux: sur l'exemple des échanges précoloniaux en pays gouro, il détruisait le mythe d'une société rurale précoloniale égalitaire en décrivant les inégalités de production, de pouvoir et de prestige entre "aînés " et "cadets ». On pourrait tout aussi bien citer la brève et stimulante réflexion (la première du genre) de Claude Tardits sur la scolarisation des filles au Dahomey (numéro 10, 1962); ou bien l'analyse de l'élaboration du pouvoir chez les Achanti « de la période de transition » par Andrezej Zajaczkowski (numéro 12, 1963), ou encore celle de Pierre Bonnafé sur la Jeunesse du mouvement national de la révolution (JMNR) comme "classe politique » dans le Congo marxiste-léniniste, une des toutes premières fois où l'on s'intéresse à la jeunesse (numéro 31, 1968); ou enfin l'article visionnaire de Peter Gutkind sur « The View from Below : Political Consciousness of the Urban Poor in Ibadan " (numéro 57, 1975), avant même le début des subaltern studies. Toutes ces analyses, et bien d'autres, remettaient en question, de façon à la fois dynamique et convaincante, l'héritage encore vivace de ce que Valentin Mudimbe $(1988,1994)$ a plus tard dénommé la «bibliothèque coloniale ». Ces textes des Cahiers ont confirmé et bercé ma formation, et celle des jeunes «africanistes » de ma génération. Je viens de les relire avec la même émotion...

6 Un autre impératif des Cahiers fut de s'ouvrir, de façon systématique, au monde anglophone. D'où la présence fréquente (même si pas obligatoire) d'un, voire de deux articles dans cette langue dans presque chaque numéro. On est encore loin $\mathrm{du}$ bilinguisme actuel, mais l'ouverture a été voulue dès l'origine. Si le premier article en anglais manque un peu d'originalité (Max Gluckman, "Tribalism in Modern British Central Africa ", numéro 1, 1960), dès le numéro 3 (il n'y a pas de texte en anglais dans le numéro 2), le tout jeune Immanuel Wallerstein fait son entrée, aux côtés du plus classique Aristide Zolberg, dans la rubrique " Sociologie politique » (sur «Ethnicity and National Integration in West Africa »). On fait place à toutes les nouveautés : Kenneth Little présente le thème alors extraordinaire en français - sauf pour Georges Balandier - de l'urbanisation africaine («Urbanization as a Social Process », numéro 3, 1960), 
thème à nouveau abordé par Peter Gutkind en 1962 dans le numéro 10 («African Urban Family Life ») et par William Bascom en 1964 dans le numéro 14 ("The Urban African and his world »); on a vu ci-dessus qu'il faudra encore dix ans pour que les Cahiers se lancent dans un numéro spécial sur le thème.

Si les anglophones sont présents dès l'origine, il faut attendre un peu pour rencontrer les premiers auteurs africains, très minoritaires. Une exception notable néanmoins : l'article d'un jeune géographe, Georges Niaxgoran-Bouah sur le village Abouré, dès le numéro 2 dirigé par Gilles Sautter, d'un jeune historien dans le numéro 7 (Romain Konka sur un établissement missionnaire au Gabon) et d'un jeune sociologue dans le numéro 8 (1962), Gomkoudougou Kabore sur les Mossi. Avant la fin des années 1960, on compte moins d'une dizaine d'auteurs africains, le plus souvent comme associés à un rapport de mission collectif - rien d'étonnant à cela, compte tenu du retard relatif pris par l'enseignement supérieur colonial français. Les exceptions notables sont, outre l'intervention plutôt de littéraires ou de linguistes, un texte de la psychiatre Solange Faladé (numéro 16, 1964), et l'article du sociologue Harris Memel-Fotê sur un guérisseur de Côte-d'Ivoire (numéro 28, 1967). Le premier article d'Ali Mazrui, politologue kenyan de renom, apparaît en 1969 (numéro 36). Au début des années 1970, il est normal que le nombre d'auteurs africains augmente sensiblement, j'en ai repéré une bonne quarantaine au total (pour autant que la consonance du nom soit révélatrice).

8 Venons-en à l'histoire dans ce tableau. A posteriori, la place de la discipline dans les Cahiers de cette époque m'a surprise : elle est très importante. Sur les 62 numéros inventoriés, 13 sont pour leur quasi totalité consacrés à l'histoire. C'est une différence majeure avec les autres disciplines: les géographes, par exemple, ont réalisé dans le même temps trois numéros spéciaux seulement et, bien que l'osmose avec les autres branches disciplinaires ait été réelle, cela ne s'est pas traduit par beaucoup de contributions, alors que, outre les 13 numéros centrés sur l'histoire (mais généralement complétés, ne serait-ce que par principe, par un ou deux textes d'autres disciplines), on rencontre au fil des pages des autres numéros au moins 24 articles complémentaires, écrits aussi bien par un historien étiqueté comme tel (Yves Person ou Claude-Hélène Perrot par exemple), que par un autre chercheur. Ainsi, le gros article du géographe Gilles Sautter sur l'histoire du chemin de fer Congo-Océan, ou celui de l'ethnologue Georges Dupré sur les Nzabi du Congo dans la traite au XIxe siècle. Au total, on arrive à quelque 80 articles, sans compter une douzaine de «notes et documents » portant sur un sujet strictement historique - et ce sans tenir compte en sus des nombreux textes qui, d'une façon ou d'une autre, comportent des éléments d'histoire. Les études traitent de tous les sujets et de toutes les périodes, depuis les temps les plus anciens jusqu'à l'époque contemporaine, même si, d'évidence, la période privilégiée par Brunschwig est celle de l'ère coloniale vécue en Afrique.

Les lecteurs des Cahiers étaient, en principe, soigneusement informés des progrès de l'histoire africaine de langue française. Henri Brunschwig tenait les choses bien en mains, et intervint personnellement ou comme responsable de numéros, à plusieurs reprises, surtout dans les premières années : on lui doit une douzaine d'articles, contre huit d'Yves Person, six de ma part, trois respectivement de Claude-Hélène Perrot et de Marc Michel, deux de Denise Bouche et de Jean-Pierre Chrétien, et un respectivement par Hélène d'Almeida-Topor, W. G. Randles et Jean-Louis Triaud. Ajoutons à cela, outre un article de fond, les régulières mises au points critiques d'Henri Moniot, 
particulièrement précieuses, intitulées selon les cas « Notes de lecture » ou « Autour de quelques livres d'histoire africaine" (l'une d'entre elles est consacrée à l'islam africain), ou encore "Histoire et cultures africaines", et même une fois "géographie africaine », enfin un point sur les historiens africains ; l'objet était de faire connaitre au lecteur le contenu et l'intérêt des ouvrages le plus souvent de sa discipline ou qui en étaient proches, tout en assurant un lien étroit avec les autres, dans un véritable esprit pluridisciplinaire : du numéro 3 (1960) au numéro 32 (1968), cette rubrique nourrie apparaît huit fois. L'équivalent n'existait guère pour les autres matières, où l'on trouve plutôt, de temps à autre, des listing bibliographiques disciplinaires. Après cette date, les Cahiers se sont mis aux comptes rendus d'ouvrages (dont la table ne commence à être donnée in extenso que dans les années 1970). En revanche, les index (par pays et, curieusement, par ethnies jusqu'en 1986) ${ }^{7}$ apparaissent dès 1964. Quant à la rubrique si utile des "ouvrages reçus", elle apparaît pour la première fois dans le numéro 37 (1970) et reste épisodique. Elle ne devient régulière qu'à partir de 1972.

10 Presque tous ceux qui allaient se faire connaître en histoire africaine sont présents ${ }^{8}$. Je n'égrène pas les noms des autres auteurs, souvent des doctorants, parfois des archivistes, sinon ceux de deux historiens traditionnels (il y en a peu de ce type) qui n'intervinrent chacun qu'une fois: Jean Valette et Robert Cornevin. La revue fit également appel à une dizaine d'historiens étrangers occidentaux (aujourd'hui comptant parmi les plus "réputés ») qui à l'époque débutaient, à la suite de Philip Curtin: Edward Alpers, Leonhard Harding, Finn Fuglestad, Richard Joseph, Joseph Miller, David Newbury, Jean-Luc Vellut, Bryan Weinstein. On ne trouve cependant aucun Britannique: sans doute parce qu'ils étaient drainés par le Journal of African History créé à Cambridge University Press la même année que les Cahiers. En revanche, l'école historique africaine (quinze articles) n'est pas en reste: Romain Konka est le premier étudiant africain de Brunschwig publié dans les Cahiers (numéro 7, 1961), suivi de deux autres mais seulement huit années plus tard (numéro 33, 1969). Quelques anglophones, nigérians ou ghanéens surtout, apparaissent, comme pour les autres disciplines, dans les années 1970. Mais les Cahiers n'ont pas saisi l'occasion de publier Joseph Ki-Zerbo (le premier Africain agrégé d'histoire) dont l'Histoire de l'Afrique noire (pourtant préfacée par Braudel) est parue en 1972. En revanche, les jeunes docteurs ivoiriens, Jean-Noël Loucou, Pierre Kipré, Semi-Bi Zan et Christophe Wondji contribuent au numéro de 1976, le dernier examiné.

11 Les Cahiers étaient, en France, à cette époque, la seule revue où l'on pouvait suivre la montée de l'histoire africaine (même si l'on ne doit pas oublier la revue interdisciplinaire Présence africaine née dès 1947, à laquelle collabora Henri Moniot à plusieurs reprises). Pourtant, entre le Centre d'études africaines et les Cahiers, la méfiance restait grande, et l'information n'a pas toujours circulé autant qu'elle l'aurait dû. Cela tenait en partie au caractère d'Henri Brunschwig, à la fois très personnel et volontiers provocateur, ce qui fit parfois interpréter sévèrement des positions pourtant raisonnables. On a, en particulier, mal lu, ou trop vite, son éditorial introduisant le premier numéro spécial Histoire des Cahiers (numéro 7, 1963), «Pour une histoire de l'Afrique noire ». De là, date l'idée fausse qu'Henri Brunschwig serait hostile à tout ce qui n'est pas source écrite. Le fait même que l'historien le plus publié dans les Cahiers soit, après lui, Yves Person, dont les sources orales furent le grand combat, infirme cette assertion (c'est dans ce même numéro 7 que Person publie son article fameux "Tradition orale et chronologie»). Brunschwig commence certes par une phrase maladroite qui fut épinglée: "Le présent cahier est consacré à l'histoire. Celle de 
l'Afrique noire est loin d'être connue, et ne le sera pas de longtemps, pour l'ensemble du continent, faute de documents écrits. On l'a dit et répété. »

Henri Brunschwig ne prend pas cette formulation à son compte, et pour cause : il est précisément en train d'introduire un numéro consacré à cette histoire, et il entend le faire en historien; ce qu'il récuse, c'est un terme qu'au même moment Hubert Deschamps (son rival en Sorbonne, il ne faut pas l'oublier) cherchait à populariser, celui d'« ethno-histoire». Ce concept avalisait en effet le credo des historiens occidentaux d'alors : pas d'histoire pour l'Afrique. Henri Brunschwig, au contraire, revendique pour l'Afrique son histoire, comme pour le reste du monde: "Le problème n'est pas de méthode [...], le problème est que, contrairement aux sociologues, les historiens ne se sont pas, jusqu'à une époque récente, intéressés à l'Afrique noire [...] ; l'ethno-histoire, qu'on pourrait définir "une histoire faite par les ethnographes", ne le résout donc pas.» Il rappelle justement que, depuis Marc Bloch, les historiens "ont, depuis longtemps, le souci d'utiliser les sources non écrites [...]. Ils se sont toujours sentis tributaires de l'archéologie et ont appris des sociologues à poser des questions et à découvrir des horizons que les anciens analystes ne soupçonnaient pas. Il est donc temps qu'enfin, les historiens, avec leurs méthodes d'historiens, exploitent les sources qui existent et, à leur tour, posent des questions aux ethnographes ». Il n'y a pas d'introduction à un numéro où il n'appelle à la collaboration entre disciplines, soulignant la nécessité pour l'historien de faire appel aux sociologues et aux ethnologues, mais réclamant aussi la réciprocité des échanges de technique, de méthode et de savoir entre spécialistes respectivement du passé et du présent. Il prônait avant tout la complémentarité des sources, quelles qu'elles soient: écrites comme orales. Il ne cachait pas son goût personnel - mais sans exclusive - pour les sources écrites: ce fut là sa provocation à une époque où le cheval de bataille de l'histoire africaine était la «tradition orale ", non sans quelque excès sur lequel on est revenu depuis lors'. Il appréciait les archives quelles que soient leur origine et leur époque, tout en reconnaissant leur insuffisance et la difficulté de les décrypter, compte tenu d'une caractéristique souvent spécifique en Afrique subsaharienne : être d'origine étrangère.

13 L'histoire africaine avait déjà commencé en Grande-Bretagne depuis une quinzaine d'années : c'est en 1947 que furent créées, à la SOAS (Université de Londres) et à l'Université de Legon au Ghana (alors encore Gold Coast), les deux premières chaires d'histoire de l'Afrique noire. Ce qui compliquait les données chez nous, c'est qu'en France on en était resté à l'histoire de la colonisation (dont Charles-André Julien occupa l'unique chaire jusqu'en 1961). L'establishment historien académique français ne voulait rien entendre; c'est un géographe, Jean Suret-Canale, communiste militant de surcroît qui, en 1958, avait publié le premier livre d'histoire de l'Afrique précoloniale. Il fallait bien qu'un maitre historien s'y mette. Ce fut le deuxième obstacle: Henri Brunschwig n'était pas un "africaniste» de formation. Il avait été spécialiste de l'histoire allemande, avant de venir à l'Afrique par l'histoire de la colonisation qu'il enseigna après-guerre à l'École coloniale (tout comme le fit Hubert Deschamps). Il s'était présenté à la succession de Charles-André Julien, qui lui avait préféré son gendre d'alors (Jean Ganiage, qui transforma le poste en chaire de démographie historique). Fernand Braudel, historien lui-même, qui avait pratiqué Brunschwig durant leur captivité commune en Allemagne, lui faisait confiance. Mais compte tenu de ce passé, pour eux un passif, les "africanistes" du Centre d'études africaines sont restés réservés. L'osmose ne se fit pas d'emblée. Georges Balandier et Gilles Sautter, le 
sociologue et le géographe, s'étaient mis d'accord pour organiser leur séminaire commun, proclamé interdisciplinaire. Brunschwig n'y participa pas. Les objectifs des uns et de l'autre étaient un peu différents : l'anthropologie africaine, de même que la géographie, l'archéologie ou la linguistique, étaient des disciplines qui avaient, toutes, pignon sur rue depuis plusieurs décennies. Il s'agissait de les rénover et de les dynamiser. La tâche de Brunschwig était un peu différente, non sans avoir quelque rapport avec le souci parallèle de Balandier de donner droit de cité à la sociologie africaine: sans rompre avec son milieu professionnel originel qui niait l'histoire de l'Afrique, Brunschwig voulait prouver à ses pairs qu'il allait contribuer à la créer. Il est probable que les réticences, voire les concurrences furent réciproques: l'historien tenait à affirmer son originalité ; il se prêtait mal au travail d'équipe ; ses collègues avaient hérité trop de mécomptes des historiens traditionnels qu'ils avaient de leur côté pratiqués. L'histoire de l'Afrique se résumait pour eux à l'histoire coloniale, et cette étiquette infamante lui resta longtemps attribuée. Au sein même des Cahiers, on peut s'interroger sur la différence établie, consciemment ou non, entre "africaniste » et «historien de l'Afrique ». Une illustration en est fournie par le premier article épistémologique de Jean Copans. En 1971, dans un texte intitulé «Pour une histoire et une sociologie des études africaines » (numéro 43), Jean Copans se concentre en fait sur l'anthropologie et la sociologie et s'intéresse à leur périodisation. Il n'exclut pourtant pas l'histoire, à laquelle il consacre un paragraphe à la deuxième page de son article : "L'histoire des études africaines est liée à l'histoire de l'Afrique (conquête coloniale, mise en valeur, décolonisation, néo-colonialisme), histoire conçue comme histoire des rapports entre l'Europe et l'Afrique, puis comme histoire des rapports entre groupes sociaux africains. »

14 Ce qui est étonnant, c'est que, à l'exception de Jean Suret-Canale dont il fait le pionnier de ce savoir repensé, Jean Copans ne fait référence à aucun historien de l'Afrique (ou d'ailleurs), ni dans le texte, ni dans la bibliographie pourtant abondante et variée qu'il donne en annexe: 44 titres dans des disciplines variées, dont par exemple Louis Althusser, Samir Amin, Jean Piager ou Julia Kristeva. C'est un peu comme s'il n'avait lu aucun des articles publiés en ce domaine dans la revue qui l'accueille pour la première fois. Cela signifie que, plus de dix ans après sa création, la communication entre chercheurs de disciplines complémentaires connaissait encore des ratés. Cette sorte de cécité vis-à-vis non de l'histoire, mais des historiens de l'Afrique a duré un peu plus que de raison, tant l'image péjorative de l'histoire coloniale avait fait des ravages... C'est heureusement de moins en moins possible; les sciences sociales sont devenues indissociables. Cela reste néanmoins une réelle tentation mono-disciplinaire, qui désormais atteint certains des historiens de l'Afrique comme les autres. En effet, dans les années 1960, s'ils voulaient échapper au carcan de l'histoire traditionnelle de la colonisation, les apprentis historiens n'avaient pas le choix : ne disposant pas encore de modèle dans leur discipline, c'est chez les autres qu'ils "trouvaient leur beurre »: parmi leurs meilleurs maîtres, on peut évoquer pour l'Afrique de l'Ouest Claude Meillassoux et Paul Pélissier, pour l'Afrique équatoriale Georges Balandier et Gilles Sautter, pour Madagascar Guy Althabe. L'histoire africaine n'aurait pu se développer aussi vite si les anthropologues, les sociologues et les géographes ne lui avaient fourni l'ensemble des matériaux et des idées qu'ils avaient collectés et disséqués depuis longtemps.

Restait, comme l'a souligné Henri Brunschwig, la méthode historique. Il témoigna en ce domaine d'une solide ténacité pour construire cette histoire de l'Afrique qui n'existait 
pas encore en France. Plusieurs de ses articles d'alors sont des morceaux d'anthologie qui, loin d'avoir vieilli, trouvent aujourd'hui une nouvelle résonance : ainsi par exemple de sa définition (on dirait aujourd'hui déconstruction) de quelques concepts, comme " colonisation", " décolonisation ", ou « indépendance ». C'est lui qui a formé presque tous les historiens francophones de l'Afrique de la génération suivante, tout en leur laissant la bride sur le cou, ce qui leur permit, les uns après les autres, de participer à l'essor de la discipline. En France sa première élève fut Denise Bouche, la deuxième moi-même et, en passant par Marc Michel et bien d'autres, son dernier fut Elikia M'bokolo. Ainsi, à la fin de sa carrière, resté sensible au fait qu'il n'avait jamais été vraiment "adopté " par les disciplines voisines, il ne s'en enorgueillissait pas moins d'avoir été, dans l'université française, le premier à faire recruter un chercheur africain.

C'est cette construction de l'histoire africaine par les Cahiers dont se félicite Pierre Alexandre dans l'avant-propos du gros numéro double 61-62 (1976), intitulé « Histoire africaine. Constatations, contestations ». Ce cahier comptait treize historiens: sept français, cinq africains et un américain, centrés sur l'histoire contemporaine, coloniale et postcoloniale. Laissons la parole au directeur de la revue à l'époque, P. Alexandre, car on ne saurait mieux conclure :

"Quand parut, voici quinze ans, le premier numéro de notre revue, certains historiens français [...] niaient la possibilité même d'une histoire scientifique de l'Afrique [...]. Le numéro spécial double que nous présentons aujourd'hui mesure le progrès accompli [...]. Nous en sommes aujourd'hui à la philosophie et à l'épistémologie de l'histoire africaine [...], c'est-à-dire au point où il vaut probablement mieux ne plus parler d'histoire africaine, mais d'histoire tout court, exercée en Afrique. »

Autrement dit, grâce aux Cahiers, Brunschwig avait réalisé son propos.

Reste une dernière question, toujours pas résolue : comment se fait-il qu'aujourd'hui encore il existe en France plusieurs revues renommées centrées majoritairement sinon exclusivement sur l'Afrique dans diverses disciplines : ethnologie et anthropologie pour la Revue des Africanistes, ou même pour L'Homme, sciences politiques (Politique africaine), alors que l'équivalent n'existe pas en histoire? Et que les revues historiennes généralistes (Les Annales, la Revue d'Histoire moderne et contemporaine, Vingtième siècle. Revue d'Histoire) commencent à peine à s'ouvrir au domaine africain, par un article de temps en temps, ou un numéro spécial relativement exceptionnel ? Les anglophones comptent depuis plusieurs décennies au moins trois revues d'histoire africaine spécialisées (outre le Journal of African History déjà cité, The International Journal of African History, et History in Africa), sans compter des revues régionales spécialisées, sur l'archéologie ou sur l'Afrique australe notamment. Les Africains francophones ont aussi depuis longtemps une revue: Afrika Zamani, le CODESRIA et même le département d'histoire de l'Université de Dakar ont lancé la leur. Les historiens français de l'Afrique n'ont rien de tel. La revue d'histoire Outre mers, enfin modernisée, couvre l'ensemble du monde moins l'Europe ; mais elle a une vie difficile, et vient en outre d'échapper à la mainmise d'une équipe désireuse de restaurer le champ de l'histoire coloniale proprement dite. La revue Afrique et Histoire s'annonçait de grande qualité : elle s'est sabordée au numéro 6 ; certes, il y a eu des querelles de personnes, mais l'éditeur aurait fait face si le marché avait suivi... Les historiens de l'Afrique sont heureux de participer à des entreprises pluridisciplinaires comme celle des Cahiers, d'être accueillis parfois par le Journal of African History ou par la Revue canadienne des Études africaines, et aussi de 
commencer à être admis dans leur propre discipline. Mais comment expliquer que, à la différence d'autres zones, le marché français de l'histoire africaine soit si étroit? Que les lecteurs français, si friands dit-on de l'histoire, soient si indifférents à celle de l'Afrique? Les conséquences sont graves, qui encouragent à corroborer dans le public (y compris chez un certain nombre d'historiens spécialistes de l'Occident) des préjugés absurdes volontiers colportés par les médias, tel celui exprimé dans le calamiteux discours prononcé par le président Sarkozy à Dakar en juillet 2007, selon lequel "l'Afrique ne serait pas encore entrée dans l'histoire "? Tout le travail réalisé par les Cahiers d'Études africaines est là pour démontrer le contraire. Continuons le combat !

\section{BIBLIOGRAPHIE}

HOBSBAWM, E. \& RANGER, T. (eds.), 1983 The Invention of Tradition, Cambridge, Cambridge University Press.

MUDIMBE, V. Y., 1988 The Invention of Africa, Bloomington, Indiana University Press.

-, 1994 The Idea of Africa, Bloomington, Indiana University Press (en cours de traduction française).

SURET-CANALE, J., 1958 Afrique noire occidentale et centrale, t. 1. Géographie, civilisations, histoire, Paris, Éditions sociales (« La culture et les hommes»).

-, 1964 Afrique noire occidentale et centrale, t. 2. L'ère coloniale, 1900-1940, Paris, Éditions sociales

(« La culture et les hommes »).

VANSINA, J., 1961 De la tradition orale, Tervuren, Musée royal de l'Afrique centrale.

-, 1985 Oral tradition as History, London, James Currey.

\section{NOTES}

1. Supposée préparer une thèse d'État sur Paris au $\mathrm{XV}^{\mathrm{e}}$ siècle (j'étais alors jeune professeure de lycée), j'ai brutalement changé d'orientation en 1960 après avoir " découvert » l'Algérie en guerre à l'occasion du service militaire à Oran de mon mari, géographe.

2. La revue ayant été fondée en 1960 , le volume 1 correspondait à l'année en cours, et le volume 2 à l'année 1961. Pour harmoniser la date au volume correspondant, un tour de passe-passe a consisté à ne dater de l'année 1962 que deux numéros (9 et 10); les deux suivants (11 et 12) sont attribués au volume 3 de 1963. En 1964, le volume 4 retrouve ses quatre numéros trimestriels (13 à 16), et ainsi de suite.

3. Selon le site des publications de l'EHESS, la revue fut créée par «Pierre Alexandre, Henri Brunschwig, Denise Paulme, Gilles Sautter, Germaine Dieterlen, Pierre Gourou, Michel Leiris", G. Dieterlen a publié un seul article (numéro 3, 1960) et les deux derniers auteurs n'apparaissent pas. Georges Balandier, qui assure le premier article du 
premier numéro, ne figurerait pas parmi les fondateurs? Il dut y avoir quelque passepasse d'ordre juridique...

4. Une des originalités du Centre d'études africaines fut alors de s'associer étroitement avec les spécialistes des langues africaines enseignant à l'École nationale des langues orientales vivantes (ENLOV).

5. J'ai été recrutée à la VI ection de l'EPHE à la rentrée 1962 (à 26 ans) en qualité de chef de travaux puis de maître-assistante (on dirait aujourd'hui maître de conférences) auprès d'Henri Brunschwig. Je suis passée à l'Université Paris-7 en 1971.

6. Les premiers articles d'historiens sur la ville africaine (Pierre Kipré et Christophe Wondji, tous deux Ivoiriens) paraissent trois ans plus tard (numéro 61-62, 1976).

7. À partir de 1987, les «Index par pays et par ethnies » sont remplacés par les «Index des pays et sociétés ».

8. Tous ces jeunes historiens se trouvaient en poste dans les universités francophones d'Afrique (y compris Françoise Raison à Madagascar), à l'exception de Henri Moniot, de W. Randles et de moi-même, avant le retour massif des coopérants à partir du début des années 1970.

9. Voir la réécriture ultérieure en 1985 (Oral Tradition as History) de l'ouvrage culte de Jan VANSINA (1961), De la tradition orale, ainsi que l'ouvrage dirigé par Eric HOBSBAWM et Terence RANGER (1983), The Invention of Tradition.

\section{RÉSUMÉS}

Résumé

Créés en 1960 avec les indépendances africaines, les Cahiers d'Études africaines furent d'emblée une revue interdisciplinaire animée par le petit groupe convaincu et novateur des directeurs d'études de cette aire culturelle. Parmi eux, Henri Brunschwig, qui refusa le concept d'ethno-histoire, assuma avec ténacité le rôle redoutable de créer le champ disciplinaire histoire de l'Afrique qui n'était alors pas reconnu par la communauté historienne dans son ensemble et guère par les autres disciplines, comme l'anthropologie ou même la géographie. Il fut en partie mal compris car la discipline, qui souffrait d'hériter de l'histoire coloniale stricto sensu, restait à inventer. On lui reprocha aussi, déformant sa pensée, d'être hostile aux sources orales. Il n'empêche : la revue contient un nombre élevé d'articles historiques, et le numéro spécial épistémologique de 1976 couronna ses efforts ; désormais le champ disciplinaire avait acquis droit de cité, couvert par des historiens professionnels aussi bien africains qu'européens.

\section{Abstract}

The Journal Cahiers d'Études africaines appeared the same year as francophone African independence occurred. It at once aimed at pluridisciplinary studies. A limited team made of convinced and audacious Directeurs d'études gathered around this new cultural area. Among them, the historian Henri Brunschwig denied any pertinence to the concept of ethno-history lauched at the time by Hubert Deschamp. Brunschwig was in charge to create the French disciplinary field of African history, at a time when neither other historians nor other social scientists, such as anthropologists or even geographers, were convinced of the possibility of writing African 
history. The former argued that written sources were lacking, the latter were defiant because of the heritage of previous colonial history. Brunschwig was partly misunderstood by his colleagues. His thoughts were partly distorted, when he was accused to despise oral sources, at a time when oral tradition was especially enhauced. In spite of all, a large number of historical special issues and other papers were published in the Journal. The 1976 special issue, focusing on epistemological questions, demonstrates that francophone African history was definitely borne and written as well by European as by African historians.

\section{INDEX}

Keywords : Pierre Alexandre, Georges Balandier, Henri Brunschwig, Denise Paulme, Gilles Sautter, Africanism, Anthropology, Ethno-History, Geography, African History, African Historians, Interdisciplinarity

Mots-clés : Pierre Alexandre, Georges Balandier, Henri Brunschwig, Denise Paulme, Gilles Sautter, africanisme, anthropologie, ethno-histoire, géographie, histoire africaine, historiens africains, interdisciplinarité

\section{AUTEUR}

\section{CATHERINE COQUERY-VIDROVITCH}

Université Paris Diderot-Paris 7, Paris. 